\title{
All-Fiber Low-Noise High-Power Femtosecond Yb-Fiber Amplifier System Seeded by an All-Normal Dispersion Fiber Oscillator
}

\author{
Pranab K. Mukhopadhyay, Kıvanç Özgören, İ. Levent Budunoğlu, and F. Ömer İlday
}

\begin{abstract}
We report an all-fiber, high-power, low-noise amplifier system seeded by an all-normal-dispersion-mode-locked Ybdoped fiber laser oscillator. Up to $10.6 \mathrm{~W}$ of average power is obtained at a repetition rate of $43 \mathrm{MHz}$ with diffraction-limited beam quality. Amplified pulses are dechirped to sub-160-fs duration in a grating compressor. It is to our knowledge the first high-power source of femtosecond pulses with completely fiber-integrated amplification comprising commercially available components. Longterm stability is excellent. Short-term stability is characterized and an integrated laser intensity noise of $<\mathbf{0 . 2 \%}$ is reported. We also conclude that all-normal dispersion fiber oscillators are low-noise sources, suitable as seed for fiber amplifiers. Detailed numerical modeling of both pulse generation in the oscillator and propagation in the amplifier provide very good agreement with the experiments and allow us to identify its limitations.
\end{abstract}

Index Terms-Femtosecond pulse propagation, optical fiber amplifiers, optical fiber lasers, ultrafast optics, Yb-doped fiber.

\section{INTRODUCTION}

$\mathbf{T}$ HERE is strong interest in the development of ultrafast fiber laser systems providing high-power femtosecond pulses increasingly robust for use in applications outside of a research laboratory. In addition to well-known applications of ultrafast lasers in areas such as nonlinear optics, metrology, and spectroscopy, numerous applications have been reported where the ultrafast laser serves as an advanced tool for material processing or imaging with subdiffraction-limit precision and greatly reduced or absent heat effects. These applications range from precision micromachining [1], and pulsed-laser deposition [2], to biomedical imaging [3], tissue processing [4], and even nanosurgery [5]. While it is clear that these emerging applications hold potential for major scientific and industrial impact, progress is hampered due to a lack of compact, lowcost, simple-to-operate, and robust laser sources. Fiber lasers can potentially address all of these requirements.

Manuscript received September 4, 2008; revised October 17, 2008 and October 28, 2008; accepted October 29, 2008. Current version published February 4, 2009. This work was supported in part by TÜBITAK under Grant 106G089 and Grant 106T017, by the DPT-UNAM Project, by EU FP7 Marie Curie IRG, by Bilkent University Research Fund PHYS-01-06, and by TÜBAGEBIP.

P. K. Mukhopadhyay, İ. L. Budunoğlu, and F. Ö. Ilday are with the Department of Physics, Bilkent University, Ankara 06800, Turkey (e-mail: pkm@ cat.ernet.in; budun@fen.bilkent.edu.tr; ilday@bilkent.edu.tr).

K. Özgören is with the Material Science and Nanotechnology Graduate Program, Bilkent University, Ankara 06800, Turkey (e-mail: kozgoren@ fen.bilkent.edu.tr).

Color versions of one or more of the figures in this paper are available online at http://ieeexplore.ieee.org.

Digital Object Identifier 10.1109/JSTQE.2008.2010261
Much progress has been made in recent years in terms of scaling up the power output of high-repetition-rate $\mathrm{Yb}$-doped fiber amplifiers using self-similar amplification [6], [7], as well as chirped-pulse amplification [8] and large-mode-area fibers (LMA) [9]. Limpert et al. [10] have demonstrated $76 \mathrm{~W}$ of average power at $75 \mathrm{MHz}$ repetition rate with $400 \mathrm{fs}$ pulse duration, and subsequently, Röser et al. [11] demonstrated average power in excess of $130 \mathrm{~W}$ with $220 \mathrm{fs}$ pulse duration. Impressive performance in terms of pulse energy has also been demonstrated up to the $1 \mathrm{~mJ}$ level at $100 \mathrm{kHz}$ repetition rate [12]. In terms of pulse duration, $70 \mathrm{fs}$ duration and $10 \mathrm{~W}$ of average power have also been obtained through the use of custom fibers and fiber-Bragg gratings for higher-order dispersion control [13]. However, these systems employ discrete optical components for coupling of the pump and signal beam to the gain fiber, and in several of the cases, a bulk solid-state oscillator to seed the amplifier, along with bulk grating stretchers. Much of the system is actually not fiber. This counters some of the main advantages of fiber. For instance, slight misalignment of the pump coupling easily leads to fiber facet damage during operation at high powers. All-fiber amplification of pulses directly from mode-locked fiber lasers has been demonstrated [14], but the results were limited to low powers due to unavailability of high pump powers.

Recently, an enabling advance has occurred. With the development of multimode pump-signal combiners (MPC), singlemode signal fiber as well as several multimode pump fibers from high-power laser diodes can be delivered to the core and cladding regions of a double-clad (DC) fiber, respectively [15]. The DC fiber can be fusion-spliced to a matching doped fiber. This approach eliminates possibility of pump misalignment.

Here, we report, to the best of our knowledge, the first demonstration of an all-fiber amplifier at the $10 \mathrm{~W}$ level with completely in-fiber delivery of the pump. The laser system is constructed using commercially available components only, and as such, it can easily be duplicated. The amplifier is seeded, also for the first time to our knowledge, by a compact allnormal-dispersion (ANDi) Yb-doped mode-locked oscillator [16]. Pulses are compressed to sub-160-fs duration using an external grating pair. We report diffraction-limited beam quality $\left(M^{2}<1.1\right)$ and intensity noise of $\sim 0.2 \%$ (measured over $20 \mathrm{~Hz}$ to $250 \mathrm{kHz}$ ) at $10.6 \mathrm{~W}$.

An all-fiber amplifier is most naturally seeded by a fiber oscillator directly from an intracavity fiber coupler [14]. Several distinct mode-locking regimes can be utilized, namely, solitonlike [17], [18], stretched-pulse [18], [19], similariton [20], and all-normal dispersion [16]. Among these, the ANDi laser does 


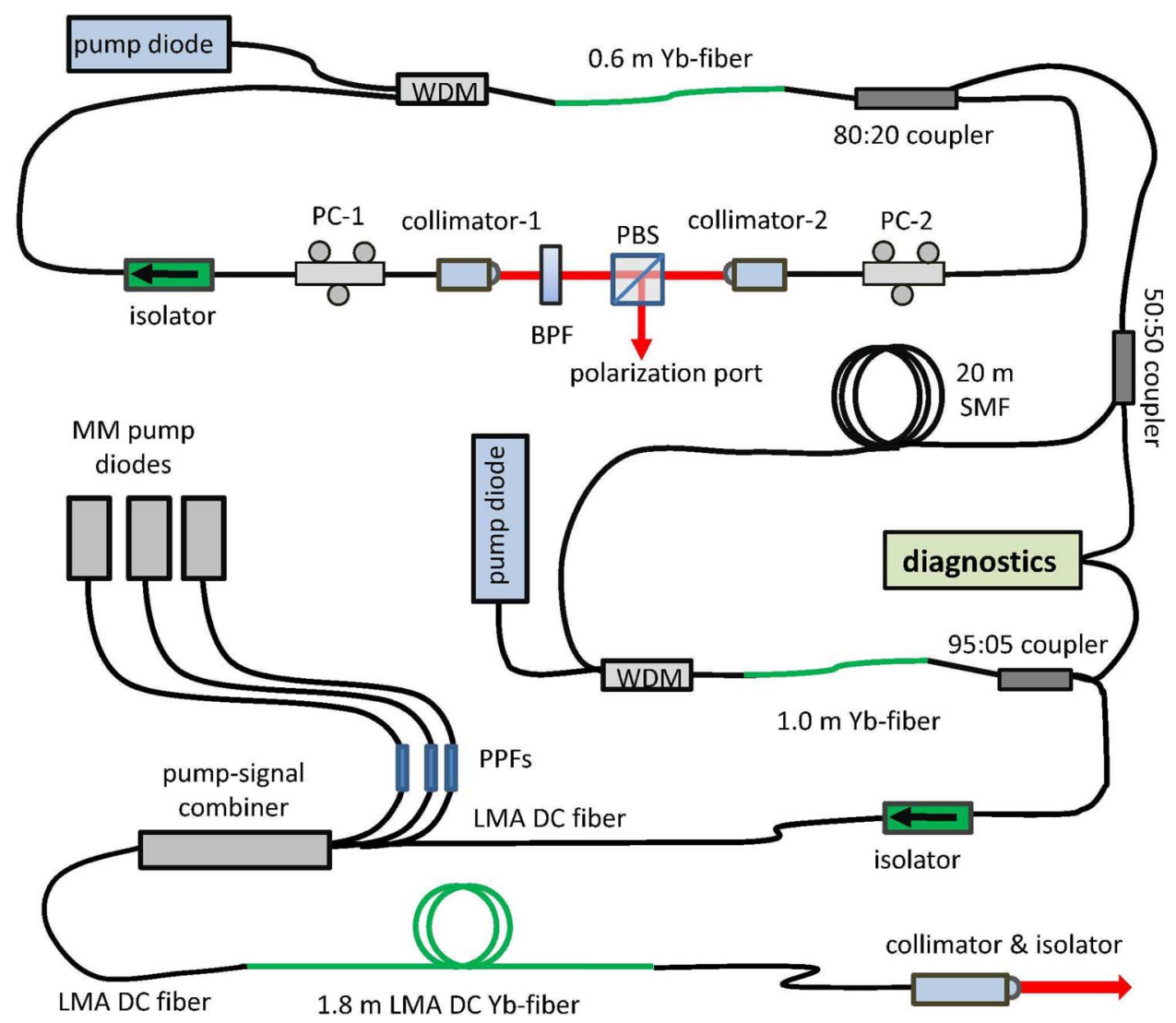

Fig. 1. Schematic of the laser setup: BPF: bandpass filter, PBS: polarizing beam splitter cube, PC: polarization controller, collimator, LMA: large-mode-area fiber, DC: double-clad fiber, SMF: single-mode fiber, PPFs: pump protection filters, WDM: wavelength-division multiplexer.

not require the use of diffraction gratings and has the potential for all-fiber integration. Owing to its simplicity and robustness, we have adapted an ANDi laser as the seed source in this study. Record-high pulse energies have been obtained from such lasers [21], [22]. On the other hand, these lasers produce relatively long pulses with residual nonlinear chirp. It was not clear to us $a$ priori whether seeding a strongly nonlinear amplifier with such pulses would not compromise the amplified pulse quality. As such, extensive numerical simulations were performed to understand the pulse shaping and guide the experiments.

\section{EXPERIMENTAL SETUP}

The experimental setup is shown schematically in Fig. 1. The system consists of the following parts: an ANDi-type Yb-doped fiber oscillator connected to a fiber pulse-stretcher followed by a preamplifier and a power amplifier. Finally, the amplified pulses are compressed in a grating compressor (not shown in the figure).

The oscillator comprises $2.1 \mathrm{~m}$ of standard single-mode fiber (SMF) for $1 \mu \mathrm{m}$ wavelength (core diameter of $6.2 \mu \mathrm{m}$, NA of 0.14 ) and $0.6 \mathrm{~m}$ of highly doped $\mathrm{Yb}$-doped gain fiber (core diameter of $6.2 \mu \mathrm{m}$, NA of $0.14, \sim 500 \mathrm{~dB} / \mathrm{m}$ absorption at $976 \mathrm{~nm}$ ), followed by another $1.7 \mathrm{~m}$ of SMF. The gain fiber is pumped in-core by a 976-nm fiber-coupled laser diode with a maximum output power of $300 \mathrm{~mW}$. Total group velocity dispersion (GVD) of the cavity is $\sim 0.1 \mathrm{ps}^{2}$. Fast saturable absorption required for mode-locking is obtained from nonlinear polarization evolution (NPE), which is implemented with two in-fiber polarization controllers and a polarizing beam splitter cube. An interference filter with $10 \mathrm{~nm}$ bandwidth is placed right after the polarizer. Due to the heavy chirp on the pulse, the filter's influence in the time domain is to remove the temporal wings of the pulse, and assist the saturable absorber. The transmission peak, centered at $1060 \mathrm{~nm}$, sets the center wavelength of the pulses [23]. An optical isolator is placed for unidirectional ring cavity configuration. Mode-locked operation is self-starting and appears to be stable indefinitely. An output power of $26 \mathrm{~mW}$ at $43 \mathrm{MHz}$ repetition rate is obtained directly through a $20 \%$ output coupler after the gain fiber. Direct fiber coupling the seed pulses to the amplifier completely eliminates the possibility of interruption of the seed due to misalignment or accidental blocking of the beam path. We found that the power from the seed laser is sufficient to suppress the amplified spontaneous emission (ASE) in the subsequent amplifying stages due to the gain peak at $1030 \mathrm{~nm}$. Around $50 \%$ of the output power from the oscillator traverses a 20-m-long SMF section to stretch the pulses. The rest is further divided for simultaneous monitoring with a fast photodiode (from which the electrical pulses are further split to a fast oscilloscope and an RF spectrum analyzer) and with an optical spectrum analyzer. Inclusion of an in-line preamplifier relaxes the power requirements from the oscillator, allowing it to be operated at moderate power levels, where its long-term operation is most stable. The pulses from the oscillator are amplified to an 
average power of $\sim 100 \mathrm{~mW}$ in the preamplifier stage consisting of a $1 \mathrm{~m}$-long $\mathrm{Yb}$-doped fiber (of the same type as in the oscillator) pumped in-core by a $976 \mathrm{~nm}$ laser diode. A small portion of the preamplifier output is split off for diagnostics and the rest seeds the power amplifier through an in-fiber optical isolator.

The power amplifier is pumped codirectionally to protect the pump diodes from optical damage due to leakage of the high-peak-power signal to the pump ports. Pump delivery to the gain fiber is accomplished with a 6-port MPC incorporating a single-mode signal feedthrough. The signal port of the MPC is an LMA fiber (core diameter of $20 \mu \mathrm{m}$, NA of 0.07 ), whereas the output of the isolator is standard SMF, resulting in a lossy, but mechanically strong splice. Due to the combined loss of the isolator $(\sim 50 \%)$ and the splice, only $25 \mathrm{~mW}$ of signal power is coupled to the output fiber of the MPC, which is an undoped DC-LMA fiber with $20 \mu \mathrm{m}$ core diameter, NA of $0.07,123 \mu \mathrm{m}$ inner cladding diameter, NA of 0.46 , and $250 \mu \mathrm{m}$ outer cladding with an octagonal structure (made of low-index polymer).

We used three $975 \mathrm{~nm}$ diodes with $8 \mathrm{~W}$ of maximum power coupled to 105 - $\mu \mathrm{m}$-diameter multimode fibers as pump for the power amplifier. Unused pump ports are utilized for monitoring the backward propagating signal. It is essential to use pump protection filters (PPF) that transmit the pump wavelength around $\sim 975 \mathrm{~nm}$ but block wavelengths above $1020 \mathrm{~nm}$ to protect the diodes from optical damage due to the feedback from the amplified signal or spontaneous emission. We measured that $85 \%$ of the pump power is coupled to the inner cladding of the output fiber of the MPC.

The high-power amplifier comprises a 1.8-m-long LMA-DC fiber with a 20- $\mu$ m-diameter, 0.07-NA ytterbium-doped core and a $125-\mu$ m octagonal-shaped cladding with NA of 0.46 . The ytterbium doping concentration is $8.5 \times 10^{25} \mathrm{~m}^{-3}$. The $\mathrm{V}$-parameter of the fiber is $\sim 4.15$, and hence, was coiled to $\sim 10 \mathrm{~cm}$ diameter to avoid the excitation of higher order spatial modes. The gain fiber is spliced with the output fiber from the MPC. The strippedoff outer cladding is recoated using a low-index polymer for uninterrupted guiding of the pump beam through the splice region. The other end of the gain fiber is spliced to a non-DC-LMA fiber to gently strip off any unabsorbed pump light. Finally, the output is taken through an in-fiber isolator-collimator with a transmission of $90 \%$. The isolator helps prevent the reflected beam from coupling back into the laser system, e.g., during material processing. For most applications, an isolator is not required.

\section{RESULTS AND DISCUSSIONS}

Numerical simulations are employed to study the pulse dynamics throughout the system, starting with pulse generation in the oscillator, followed by propagation through the fiber strecher, preamplifier, power amplifier, and grating compressor (Fig. 1). We run separate simulations for pulse generation in the oscillator and pulse propagation outside the oscillator. The numerical output of oscillator simulation serves as the initial condition for propagation through the fiber stretcher, the preamplifier, the power amplifier, and the grating compressor.
Pulse propagation in each medium is modeled with nonlinear Schrödinger equation generalized to include third-order dispersion (TOD), gain with saturation, and frequency dependence and intrapulse Raman scattering [20], [24]

$$
\begin{aligned}
\frac{\partial A}{\partial z} & +i \frac{\beta^{(2)}}{2} \frac{\partial^{2} A}{\partial \tau^{2}}-\frac{\beta^{(3)}}{6} \frac{\partial^{3} A}{\partial \tau^{3}}=\frac{g}{2} A+\delta \frac{\partial^{2} A}{\partial \tau^{2}} A \\
& +i \gamma|A|^{2} A+i \gamma T_{R} \frac{\partial|A|^{2}}{\partial \tau} A
\end{aligned}
$$

where $A(z, \tau)$ is the slowly varying pulse envelope in a retarded time frame $(\tau), z$ is the propagation coordinate, $\beta^{(2)}$ is the GVD parameter, $\beta^{(3)}$ is the TOD parameter, $\gamma$ is the cubic nonlinearity of the fiber section given by $\gamma=n_{2} \omega_{0} / c A_{\text {eff }}$, where $n_{2}$ is the Kerr coefficient, $\omega_{0}$ is the central angular frequency, $c$ is the velocity of light in vacuum, and $A_{\text {eff }}$ is the effective beam area, $T_{R}$ is related to the slope of the Raman gain spectrum, which is assumed to vary linearly with frequency around $\omega_{0}, g$ is the net gain, and $\delta$ is related to the gain bandwidth in the parabolic frequency dependence approximation. $g$ and $\delta$ are nonzero only for the doped fibers. Gain saturates with total energy according to

$$
g(E)=g_{\mathrm{ss}} /\left(1+\int|A|^{2} d \tau / E_{\mathrm{sat}}\right)
$$

where $g_{\mathrm{ss}}$ is the small-signal gain. The gain bandwidth is taken to be $40 \mathrm{~nm}$ [24], [25], $E_{\mathrm{sat}}$ is the gain saturation energy. The governing equation is solved by the standard split-step method in each medium.

The following are the parameters used for the oscillator fiber segments: $\beta^{(2)}=23 \mathrm{fs}^{2} / \mathrm{mm}, \beta^{(3)}=41 \mathrm{fs}^{3} / \mathrm{mm}, n_{2}=$ $2.3 \times 10^{-16} \mathrm{~cm}^{2} / \mathrm{W}$; the effective mode area for the SMF was found to be $30 \mu \mathrm{m}^{2}$ and that for the gain fiber is $22 \mu \mathrm{m}^{2}$. The fiber is followed by a lumped saturable absorber that represents NPE and a Gaussian-shaped bandpass filter with $10 \mathrm{~nm}$ bandwidth (both of which are modeled as transfer functions, in time and frequency domains, respectively). The initial field is white noise. Simulations are run until the field converges within a finite number of round trips through the cavity. We verify numerical accuracy by checking that the results are unchanged after doubling each of the sampling resolutions. In Fig. 2(a) and (b) we summarize the simulation results for the oscillator with total cavity GVD of $0.1 \mathrm{ps}^{2}, E_{\mathrm{sat}}=1.8 \mathrm{~nJ}$, and $g_{0}=$ $30 \mathrm{~dB}$. The lengths and positioning of the fibers are as used in the experiments. The combined action of gain, nonlinearity, normal dispersion, and spectral filtering leads to linearly chirped optical pulses with a spectral profile that develops the steep and structured edges characteristic of self-phase modulation (SPM). Starting at the location of collimator-1, the full-width at halfmaximum (FWHM) temporal and spectral widths of the pulse both increase monotonically in the SMF section [Fig. 2(b)]. In the gain fiber and the second SMF section, the spectral width remains nearly constant owing to gain narrowing in the $\mathrm{Yb}$ fiber and increased pulse duration in the second SMF section, but the pulse duration continues to increase due to GVD. We find that NPE has relatively little effect, compared to the spectral filter terms of their effect on the temporal and spectral widths. These findings are in agreement with those of references [16], [21]. As 


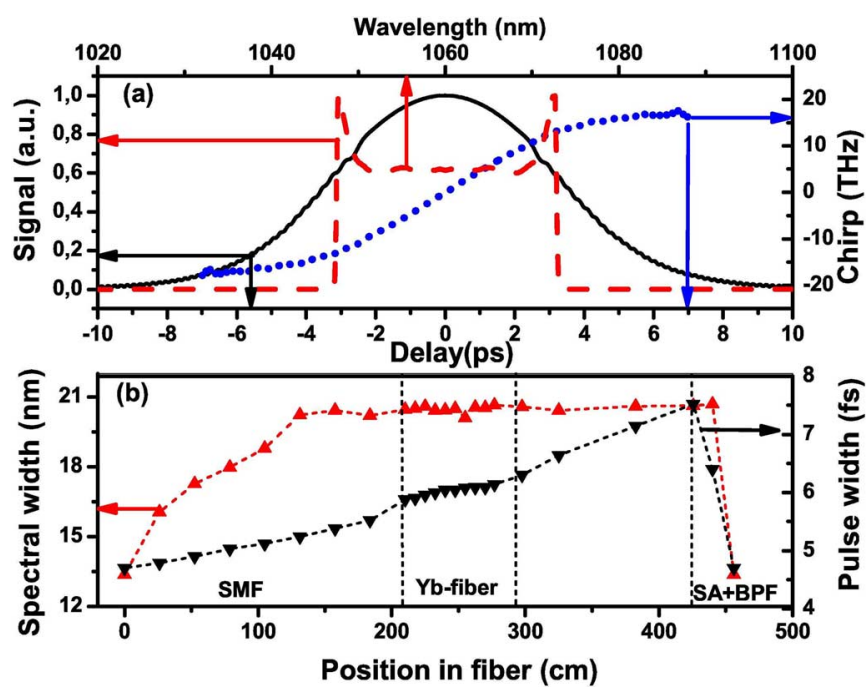

Fig. 2. Numerical simulation results for the oscillator: (a) Pulse characteristics at the $20 \%$ output port, showing the temporal shape (black, solid line), the spectrum (red, dashed line), and temporal chirp (blue, dotted line). (b) Variation of the spectral (red, upward triangles) and temporal (black, downward triangles) width (FWHM) along the length of the cavity (referenced to collimator-1. Lines are to only guide the eye.

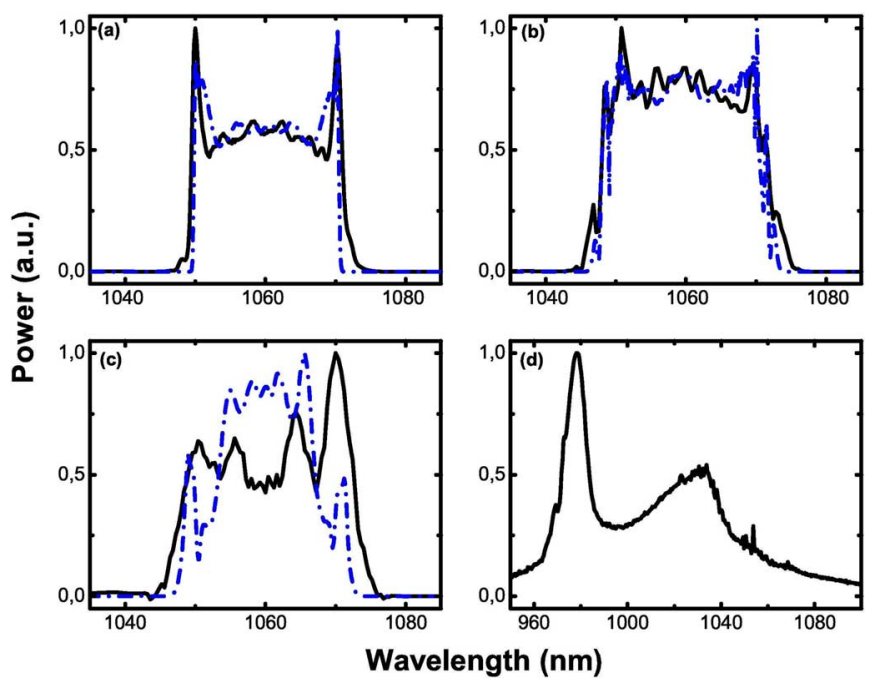

Fig. 3. Measured (solid, black lines) and simulated (dash-dotted, blue lines) spectra obtained from: (a) oscillator output, (b) preamplifier output, (c) amplifier output (at $10.6 \mathrm{~W}$ of power), (d) an unused pump port (showing backwardpropagating ASE signal and residual pump power).

shown in Fig. 2(a), though the spectrum is highly structured, the corresponding temporal profile is a smooth, positively chirped Gaussian with 7 ps FWHM width at the fiber-coupled output of the oscillator (taken as input for the propagation simulations). Width of the chirped pulses from the polarization port inferred from autocorrelation measurements agree very well with the numerical results. Further, the simulated and experimentally measured spectra for the oscillator output match very closely [Fig. 3(a)]. The bandwidth is $\sim 22 \mathrm{~nm}$, corresponding to a transform-limited pulse width of $132 \mathrm{fs}$.

The pulses from the oscillator are stretched in a 20 -m-long SMF section. Based on the numerical simulations, after the stretcher fiber, the pulse duration increases to $\sim 18 \mathrm{ps}$. The stretched pulses are amplified in a 1-m-long single-mode $\mathrm{Yb}$ fiber. The measured and simulated spectra of the pulses after the preamplifier are shown in Fig. 3(b). The spectral width is increased slightly to $23.5 \mathrm{~nm}$ and some oscillations develop at the edges of the spectrum due to nonlinearity. There is no significant ASE signal at the gain peak of $1030 \mathrm{~nm}$, nor a Raman-shifted signal at longer wavelengths. For the simulations, we adjust the saturation energy of the gain fiber to obtain the same amplification factor as in the experiment.

Pulse propagation through the power amplifier is simulated in the same manner, with the effective mode area of the gain fiber set to $314 \mu \mathrm{m}^{2}$ and the length is $1.8 \mathrm{~m}$. The gain in the simulations is adjusted to obtain the same amplification factor of the pulse energy for the corresponding pump power in the experiments. The main features of the simulated and experimental spectra match very well at low powers. Fig. 3(c) shows results at $10.6 \mathrm{~W}$, where the agreement is still reasonable. At $10.6 \mathrm{~W}$ of average power, the pulse energy is $\sim 230 \mathrm{~nJ}$ and the peak power is estimated to be $13 \mathrm{~kW}$. Some oscillations develop on the spectrum only at high powers, which we attribute to polarization beating. In addition, the oscillations are accompanied by quick variation of the polarization state with pump power. This can be avoided with a polarization-maintaining fiber system. Fig. 3(d) shows a spectrum recorded from an unused pump port of the MPC, showing a small amount of (few milliwatts) backward-propagating ASE and residual pump light. The backward-propagating light contains a small fraction of the pulses as well. For this reason, protection of the pump diodes is essential.

In order to better understand the power scaling characteristics and ASE production in the power amplifier, we model amplification of the average signal power as a three-level amplifying medium. Gain and loss for pump and signal at a given longitudinal position $z$ along the fiber is given by the equations [25], [26]

$$
\begin{aligned}
& \frac{d P_{p}}{d z}=\eta_{p}\left(\sigma_{e p} n_{2}-\sigma_{a p} n_{1}\right) N_{\text {tot }} P_{p} \\
& \frac{d P_{s}}{d z}=\eta_{s}\left(\sigma_{e s} n_{2}-\sigma_{a s} n_{1}\right) N_{\text {tot }} P_{s}
\end{aligned}
$$

where $P_{p}$ and $P_{s}$ are the pump power and the average signal power, respectively; $\eta_{p}$ and $\eta_{s}$ are the overlap factors for the pump and signal, respectively; $\sigma_{e p}$ and $\sigma_{a p}$ are the emission and absorption cross sections for the pump beam, and $\sigma_{e s}$ and $\sigma_{a s}$ are those for the signal wavelength. $N_{\text {tot }}$ is the total population density and $n_{1}$ and $n_{2}$ are the population densities for the lower and upper levels, respectively. The upper- and lower-state population densities have steady-state values given by

$$
n_{2}=\frac{R_{12}+W_{12}}{R_{12}+R_{21}+W_{12}+W_{21}+A_{21}}, \quad n_{1}=1-n_{2} .
$$

Here $A_{21}$ is the spontaneous transition rate. The transition rates are given by:

$$
\begin{array}{ll}
R_{12}=\sigma_{a p} I_{p} / h \nu_{p}, & R_{21}=\sigma_{e p} I_{p} / h \nu_{p} \\
W_{12}=\sigma_{a s} I_{s} / h \nu_{s}, & W_{21}=\sigma_{e s} I_{s} / h \nu_{s}
\end{array}
$$




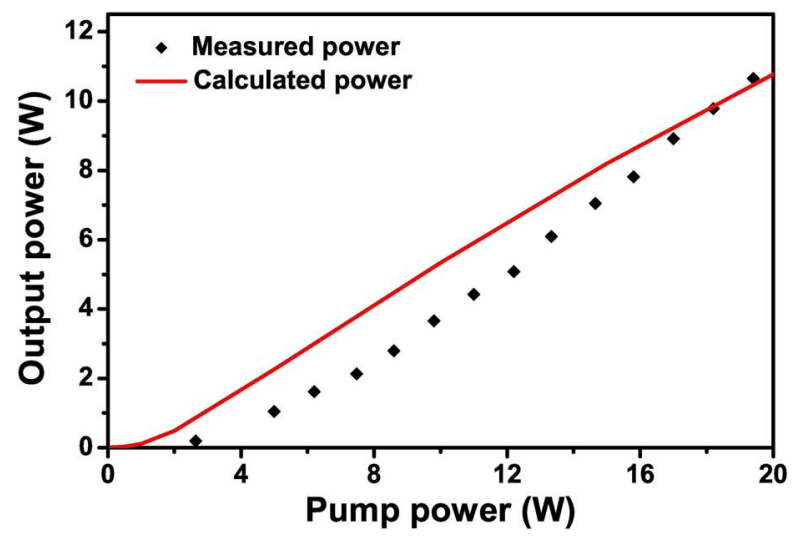

Fig. 4. Power scaling characteristics of the power amplifier. Points represent measured output power versus total pump power. Solid line shows the simulation results.

where $I_{i}\left(h \nu_{i} / \tau_{f} \sigma_{i}\right)_{(i=p, s)}$ represents the saturation intensity for the pump and signal wavelengths, $\nu_{i}$ is the corresponding frequency, and $\tau_{\mathrm{f}}$ is the upper-state lifetime. The following values are used: core diameter, $20 \mu \mathrm{m}$; cladding diameter, $125 \mu \mathrm{m}$; fiber length, $1.8 \mathrm{~m} ; N_{\text {tot }}=8.5 \times 10^{25} \mathrm{~m}^{-3} ; \sigma_{a p}=$ $25 \times 10^{-13} \mu \mathrm{m}^{2}, \quad \sigma_{e p}=21.7 \times 10^{-13} \mu \mathrm{m}^{2}, \quad \sigma_{a s}=0.448 \times$ $10^{-13} \mu \mathrm{m}^{2}, \sigma_{e s}=2.62 \times 10^{-13} \mu \mathrm{m}^{2}$, and $\tau_{f}=0.8 \mathrm{~ms}$. Experimentally measured and calculated signal power dependences on pump power are shown in Fig. 4. At a total of $20 \mathrm{~W}$ of coupled pump power, we measure $10.6 \mathrm{~W}$ at the isolator-collimator port, corresponding to $53 \%$ optical conversion efficiency. We attribute deviation of the measured values from calculations at lower pump powers to a shift of the diode emission wavelength. At low pump powers, the peak emission wavelength from the diodes is around $955-960 \mathrm{~nm}$, which is far away from the peak absorption wavelength $(\sim 976 \mathrm{~nm})$ in Yb-doped fiber. This can be compensated by increasing the diode temperature, which was avoided not to strain the diodes. As the drive current is increased, the emission wavelength shifts toward $976 \mathrm{~nm}$.

Chirped pulses from the power amplifier are compressed to near-transform limit in a grating pair in near-Littrow configuration and characterized with short-span interferometric and longspan intensity autocorreletions. The overall efficiency of the current grating compressor is around 50\%. However, efficiencies as much as $80 \%$ are readily available with higher quality gratings. An interferometric autocorrelation (AC) trace of the dechirped pulses corresponding to $10.6 \mathrm{~W}$ of average power is shown in Fig. 5. The FWHM width of the AC is $240 \mathrm{fs}$, which corresponds to an estimated pulse duration of $\sim 160$ fs (assuming a Gaussian pulse shape). Despite the highly structured spectral shape, the temporal profile is reasonably smooth. The side lobes contain less than $10 \%$ of the pulse energy. The inset of Fig. 5 shows the corresponding long-range intensity AC trace. Possibility of multiple pulsing is eliminated by carefully checking that: i) the long-range $\mathrm{AC}$ trace shows no secondary pulse (up to $16 \mathrm{ps}$ range, obtained by offsetting zero-delay point); ii) the RF pulse spectrum is not modulated (using a $12 \mathrm{GHz}$ photodiode and a $26 \mathrm{GHz}$ spectrum analyzer, thus eliminating a secondary pulse with temporal spacing of $>25 \mathrm{ps}$ ); and iii) ensuring absence

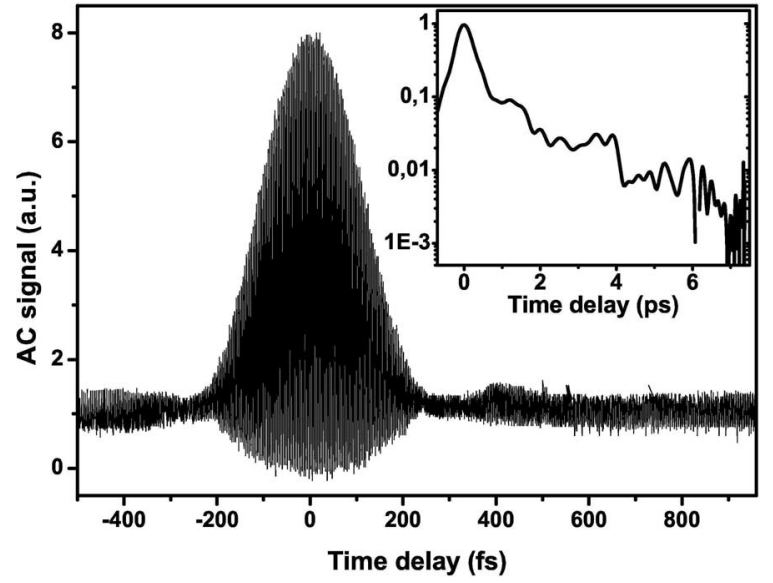

Fig. 5. Interferometric autocorrelation trace of dechirped pulses at $10 \mathrm{~W}$ of power. Inset shows long-range intensity autocorrelation on semilog scale.

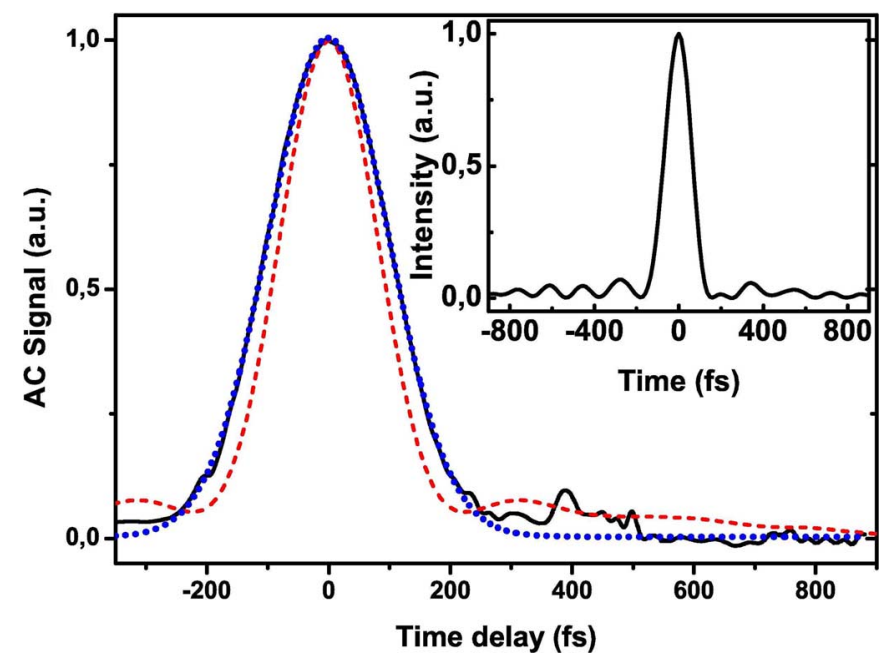

Fig. 6. Intensity autocorrelation of the measured (solid, black line), along with a Gaussian fit (dotted, blue line) and simulated (dashed, red line) pulses at $10 \mathrm{~W}$ of power. Inset shows the corresponding simulated temporal pulse shape.

of periodic modulation on the optical spectrum (wavelength resolution of $15 \mathrm{pm}$ of the analyzer corresponds to absence of a secondary pulse within a time window of $<200 \mathrm{ps}$ ). The autocorrelation of the simulated temporal shape is calculated based on simulated grating compression with corresponding GVD and TOD parameters and the grating spacing optimized for minimum chirp. The FWHM width of the simulated pulse is $148 \mathrm{fs}$, in close agreement with the experiment. The intensity $\mathrm{AC}$ traces of the measured and the simulated pulses are plotted in the Fig. 6, showing reasonable agreement. The simulated pulse shape is shown in the inset. The measured pulse duration at $10 \mathrm{~W}$ is $25 \%$ higher than the zero-phase-transform-limited value, which we attribute to uncompensated TOD and SPM.

The measured and simulated variation of the FWHM pulse width as a function of the output power is shown in Fig. 7. Initially, the pulse width decreases with power as SPM-induced spectral broadening counteracts gain narrowing. The minimum pulse duration of $137 \mathrm{fs}$ is obtained at $5 \mathrm{~W}$ of power. However, beyond this point, the pulse width gradually increases due to 


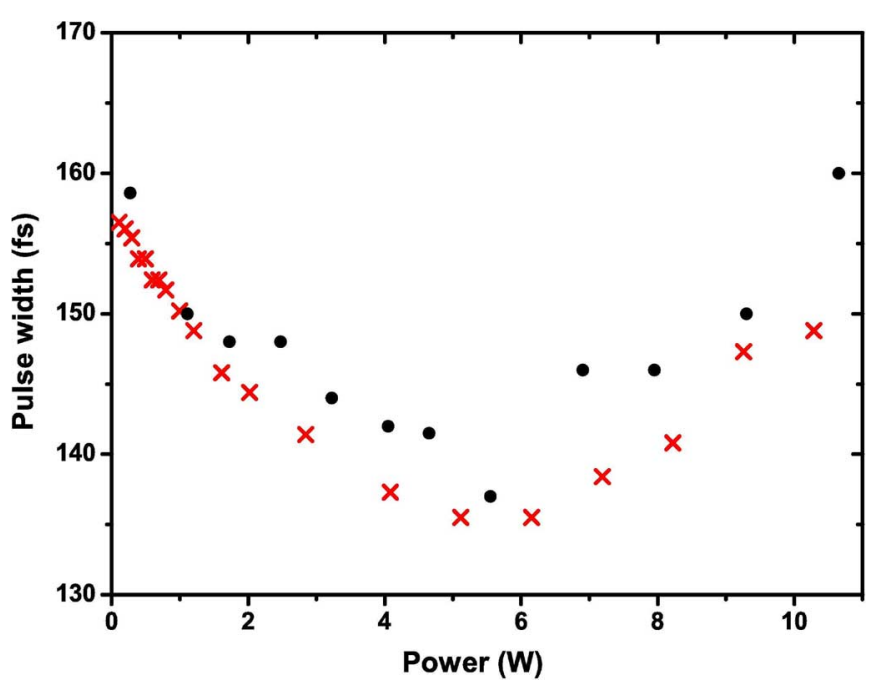

Fig. 7. Measured (black circles) and simulated (red crosses) variation of the FWHM width of the compressed pulse as a function of the output power.

excessive SPM. The optimal amount of grating spacing required to dechirp the pulses over the range of operating power corresponds to a total GVD range of $-0.93 \mathrm{ps}^{2}$ to $-0.97 \mathrm{ps}^{2}$. The amount of TOD from the gratings over the same range is about $+0.00015 \mathrm{ps}^{3}$. The TOD introduced by the grating pair is of the same sign as that of the optical fiber. This indirectly places a limitation on the maximum pulse energy achievable, since further stretching of the pulses to reduce SPM would lead to accumulation of even more TOD in the compressor. It has been shown that prisms can be introduced in the pulse compressor for TOD control [27].

Good beam quality is essential for any intended application and it must be checked carefully when using LMA fibers that are not strictly single mode. The spatial beam quality of the laser system is characterized by the well-known beam propagation method [28]. To this end, an artificial waist is created by focusing the beam and the beam radius is measured around the waist region as a function of distance from a reference plane with a beam profiler and motorized delay stage. The $M^{2}$ parameter is estimated using the propagation equation given by

$$
w(z)=w_{0} \sqrt{1+\left[\frac{M^{2} \lambda\left(z-z_{0}\right)}{\pi w_{0}^{2}}\right]^{2}}
$$

where $w(z)$ is the spot radius at a distance $z$ from the reference plane, $w_{0}$ the waist radius, $\lambda$ the wavelength of the beam, $z_{0}$ is the waist location and $M^{2}$ is the beam propagation factor. In Fig. 8, we plot the variation of $M^{2}$ parameter as a function of the output power. It can be seen that the beam quality remains close to the diffraction-limited value $\left(M^{2}<1.1\right)$ even at $10.6 \mathrm{~W}$ of power. The estimated error for the $M^{2}$ value obtained by fitting of the multimode beam propagation equation is $<3 \%$. The beam is circular in shape with a Gaussian intensity distribution (inset of the Fig. 8).

The signal-to-noise ratio of nearly all laser experiments is ultimately limited by fluctuations in the laser power, i.e., laser intensity noise. Low laser intensity noise is directly linked to

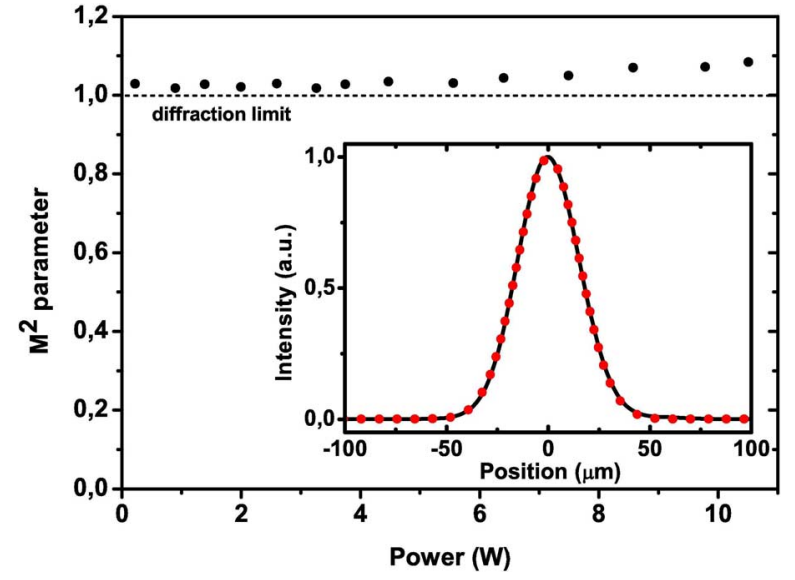

Fig. 8. Variation of the $M^{2}$ parameter with output power. Inset is a measured beam profile (solid, black line) along with a Gaussian fit to it (red, dotted line).

repeatability and uniformity in applications based on lasermaterial interactions with femtosecond pulses due to the highly nonlinear nature of these interactions. It is well known that intensity noise can easily be increased during amplification due to pump noise of the amplying stages and ASE introduced during amplification, which can be kept low through careful optimization [29]. Even though impressive advances have been made with ever more powerful Yb-doped fiber amplifiers in recent years, there is virtually no discussion of how the noise is effected in the high-power systems. Given that multiple stages of amplification are used and the system is typically optimized for maximum power output, there is reason to think that excess noise may be introduced. Any fluctuations due to asynchronous shift of the wavelengths with the pump power and temperature of the diodes can lead to increase in intensity noise. Therefore, we characterized the relative intensity noise (RIN) of our laser system using the standard method [30]. In this method, the laser signal is detected with an InGaAs with 5 ns rise time. Since the detected periodic signal has an amplitude modulation spectrum common to all harmonics, measuring the spectrum of the photocurrent at baseband gives the desired noise spectrum. Signal at baseband is obtained by passing the photocurrent through a low-pass filter (dc to $1.9 \mathrm{MHz}$ ). The output of the filter is sent to a high dynamic range baseband spectrum analyzer (bandwidth of $250 \mathrm{kHz}$ ) to obtain the noise spectrum. The noise spectrum gives the RIN in the photocurrent signal at the single sideband (SSB). Fig. 9(a) displays the SSB spectral density of RIN of the output from the oscillator-amplifier system. It can be seen from the figure that the noise is increased significantly in the amplifier stage at high powers. It is convenient to refer to the integrated noise for quantitative comparison, which is obtained by integrating the spectral density of RIN over a specified bandwidth (also multiplied by a factor of 2 for double sidebands). Fig. 9(b) shows the calculated amplitude noise of the amplifier as a function of output power, integrated over a frequency range from $20 \mathrm{~Hz}$ to $250 \mathrm{kHz}$. The integrated noise of the oscillator is as low as $0.029 \%$ over the same range. Integrated noise for the amplifier output starts from $0.06 \%$ at lower powers and increases up to $\sim 0.2 \%$ at $10 \mathrm{~W}$. Systematic studies are needed to fully 


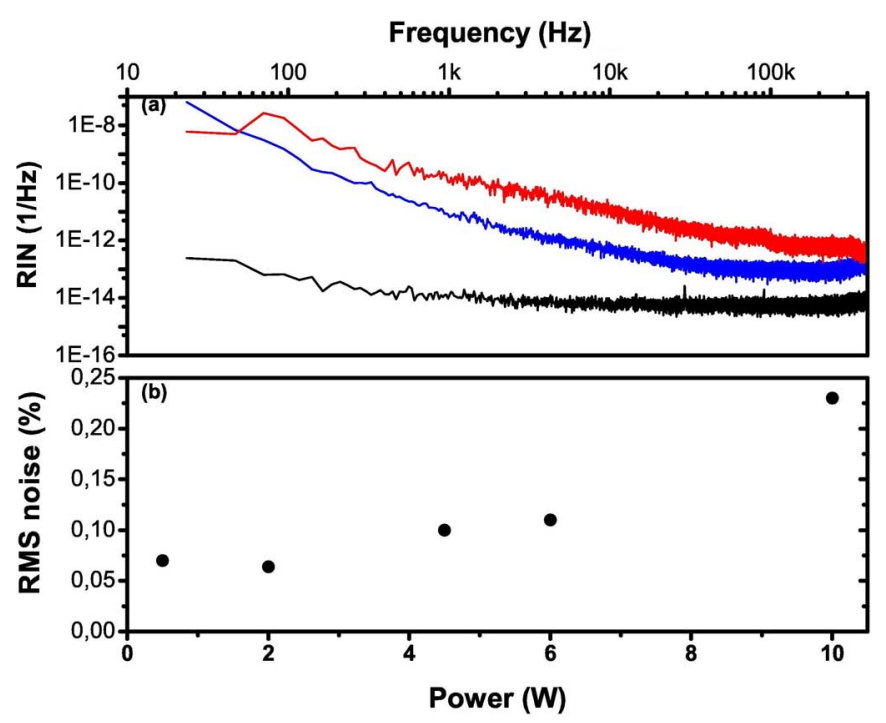

Fig. 9. (a) Measurement of relative intensity noise (RIN): upper (red) curve is the amplifier RIN at $10 \mathrm{~W}$ of power, middle (blue) curve is the oscillator RIN, and lower (black) curve is the noise floor. (b) Variation of integraterd noise as a function of power for the frequency range of $20-250 \mathrm{kHz}$. Integrated noise of the oscillator is $0.029 \%$ over the same range.

understand the reason of the increase, but we attribute it partly to the small amount of ASE generated during amplification and partly to the better wavelength stability of the single-mode oscillator pump diode compared to the high-power pump diodes. To the best of our knowledge, this is the first time that the intensity noise of an ultrafast fiber-based amplifier system has been characterized. The noise level is found to be comparable to lownoise continuous-wave fiber lasers [31] and significantly better than bulk ultrafast solid-state lasers.

We have explored the limitations of the present system through experiments and simulations. In terms of average power, higher levels are possible and we have obtained up to $15 \mathrm{~W}$ by adding a 4th pump diode. However, we have had failures due to pump power leaking into the coating region and causing damage. This appears to be a limitation of the particular fiber we have used in our experiments. The pulse energy is currently limited by nonlinearity; higher energies would lead to distortion of the pulse shape. This can be overcome by reducing the undoped lead fiber of the collimator, by further chirping the pulses together by implementing TOD control [27] or by utilizing SPM-TOD compensation [32], [33]. The latter, however, requires use of several hundred meters of stretcher SMF. Pulse duration is currently limited by TOD and SPM. However, given the pulse quality available from an ANDi laser and in the presence of gain narrowing, improvement beyond $\sim 25 \%$ seems difficult unless nonlinear pulse compression is utilized. Finally, it should be possible to reduce the amplifier intensity noise to values comparable to the oscillator noise by even better suppression of the small amount of ASE introduced in the power amplifier.

We have succesfully used this laser system in our laboratory for processing of various materials, primarily modification of $\mathrm{Ti}$ disk surfaces for biomedical applications.
These results demonstrate the utility of the laser system and constitute an independent confirmation of the beam and pulse quality. These findings will be reported in a separate platform.

\section{CONCLUSION}

In conclusion, we have demonstrated a $10 \mathrm{~W}$ fiber amplifier, based on strictly in-fiber pump delivery from several multimode diodes via a multimode pump-signal combiner. The compact laser system consists of commercially available components, and is compact and robust. Pump-to-signal optical efficiency is $53 \%$. The amplifier is seeded by pulses from an ANDi-type fiber laser for the first time. The repetition rate is $43 \mathrm{MHz}$. Amplified pulses are compressed to sub-160-fs duration, which shows that ANDi-type fiber lasers are suitable seed lasers, offering a simpler cavity structure and great potential for all-fiber integration. Nearly diffraction-limited $\left(M^{2}<1.1\right)$ beam quality is obtained even at the highest powers. Despite its importance for virtually all applications, intensity noise of high-power femtosecond fiber amplifiers is largely unknown. As a first step in this direction, we have characterized the noise of the present system, which is measured to be $<0.2 \%$. We measured the ANDi-type oscillator's intensity noise to be $<0.03 \%$. Extensive numerical simulations explain the observed behavior of the system and closely guide the experimental efforts.

Note added in proof: We have recently obtained stable operation with up to $20 \mathrm{~W}$ of average power by replacing the original Yb-doped fiber with a new segment of Yb-doped fiber of the same type and adding an additional pump diode.

\section{REFERENCES}

[1] S. Nolte, "Micromachining," in Ultrafast Lasers, M. E. Fermann, A. Galvanauskas, and G. Sucha, Eds. New York: Marcel Dekker, 2003, pp. 359-394.

[2] E. G. Gamaly, A. V. Rode, and B. Luther-Davies, "Ultrafast laser ablation and film deposition," in Pulsed Laser Deposition of Thin Films, R. Eason, Ed. Hoboken, NJ: Wiley, 2007, pp. 99-130.

[3] W. Denk, J. H. Strickler, and W. W. Webb, "Two-photon laser scanning fluorescence microscopy," Science, vol. 248, pp. 73-76, 1990.

[4] H. Lubatschowksi and A. Heisterkamp, "Interaction with biological tissue," in Femtosecond Technology for Technical and Medical Applications, F. Dausinger, F. Lichtner, and H. Lubatschowki, Eds. Heidelberg, Germany: Springer-Verlag, 2004, pp. 91-104.

[5] A. Vogel, J. Noack, G. Hütman, and G. Paltauf, "Mechanisms of femtosecond laser nanosurgery of cells and tissues," Appl. Phys. B, vol. 81, pp. 1015-1047, 2005.

[6] M. E. Fermann, V. I. Kruglov, B. C. Thomsen, J. M. Dudley, and J. D. Harvey, "Self-similar propagation and amplification of parabolic pulses in optical fibers," Phys. Rev. Lett., vol. 84, pp. 6010-6013, 2004.

[7] J. Limpert, T. Schreiber, T. Clausnitzer, K. Zöllner, H.-J. Fuchs, E.-B. Kley, H. Zellmer, and A. Tünnermann, "High-power femtosecond Yb-doped fiber amplifier," Opt. Express, vol. 10, pp. 628-638, 2002.

[8] D. Strickland and G. Mourou, "Compression of amplified chirped optical pulses,” Opt. Commun., vol. 56, pp. 219-221, 1985.

[9] N. G. R. Broderick, H. L. Offerhaus, D. J. Richardson, and R. A. Sammut, "Power scaling in a passively mode-locked large-mode area fiber laser," IEEE Photon. Technol. Lett., vol. 10, no. 12, pp. 1718-1720, Dec. 1998.

[10] J. Limpert, T. Clausnitzer, A. Liem, T. Schreiber, H. J. Fuchs, H. Zellmer, E. B. Kley, and A. Tunnermann, "High-average-power femtosecond fiber chirped-pulse amplification system," Opt. Lett., vol. 28, pp. 1984-1986, 2003.

[11] F. Röser, J. Rothhard, B. Ortac, A. Liem, O. Schmidt, T. Schreiber J. Limpert, and A. Tünnermann, " $131 \mathrm{~W} 220$ fs fiber laser system," Opt. Lett., vol. 30, pp. 2754-2756, 2005. 
[12] F. Röser, T. Eidam, J. Rothhardt, O. Schmidt, D. N. Schimpf, J. Limpert, and A. Tünnermann, "Millijoule pulse energy high repetition rate femtosecond fiber chirped-pulse amplification system," Opt. Lett., vol. 32, pp. 3495-3497, 2007.

[13] T. R. Schibli, I. Hartl, D. C. Yost, M. J. Martin, A. Marcinkevicius, M. E. Fermann, and J. Ye, "Optical frequency comb with submillihertz linewidth and more than $10 \mathrm{~W}$ average power," Nat. Photon., vol. 2, pp. 55-59, 2008.

[14] F. O. Ilday, H. Lim, J. R. Buckley, and F. W. Wise, "Practical all-fiber source of high-power, $120 \mathrm{fs}$ pulses at $1 \mu \mathrm{m}$," Opt. Lett., vol. 28, pp. 13621364, 2003.

[15] F. Gonthier, L. Martineau, N. Azami, M. Faucher, F. Seguin, D. Stryckman, and A. Villeneuve, "High-power all-fiber components: The missing link for high power fiber lasers," Proc. SPIE, vol. 5335, pp. 266-276, 2004.

[16] A. Chong, J. Buckley, W. Renninger, and F. W. Wise, "All-normaldispersion femtosecond fiber laser," Opt. Express, vol. 14, pp. 1009510100, 2006.

[17] I. N. Duling, III, "Subpicosecond all-fiber erbium laser," Electron. Lett., vol. 27, pp. 544-545, 1991.

[18] H. Lim, F. O. Ilday, and F. W. Wise, "Generation of 2-nJ pulses from a femtosecond fiber laser," Opt. Lett., vol. 28, pp. 660-662, 2003.

[19] K. Tamura, E. P. Ippen, H. A. Haus, and L. E. Nelson, "77-fs pulse generation from a stretched-pulse mode-locked all-fiber ring laser," Opt. Lett., vol. 18, no. 13, pp. 1080-1082, 1993.

[20] F. O. Ilday, J. R. Buckley, W. G. Clark, and F. W. Wise, "Self-similar evolution of parabolic pulses in a laser," Phys. Rev. Lett., vol. 92, no. 21, pp. 213902-1-213902-4, 2004.

[21] A. Chong, W. Renninger, and F. W. Wise, "All-normal-dispersion femtosecond fiber laser with pulse energy above $20 \mathrm{~nJ}$," Opt. Lett., vol. 32, pp. 2408-2410, 2007.

[22] J. An, D. Kim, J. W. Dawson, M. J. Messerly, and C. P. Barty, "Gratingless, fiber-based oscillator that generates $25 \mathrm{~nJ}$ pulses at $80 \mathrm{MHz}$, compressible to 150 fs," Opt. Lett., vol. 32, pp. 2010-2012, 2007.

[23] H. Lim, J. R. Buckley, and F. W. Wise, "Wavelength tunability of femtosecond Yb fiber lasers," in Proc. Conf. Lasers Electro-Opt. (CLEO 2004), May $16-21$, vol. 2, pp. 2-3.

[24] F. O. Ilday, H. Lim, J. R. Buckley, F. W. Wise, and W. G. Clark, "Generation of 50-fs, 5-nJ pulses at $1.03 \mu \mathrm{m}$ from a wave-breaking-free fiber laser," Opt. Lett., vol. 28, pp. 1365-1367, 2003.

[25] R. Paschotta, J. Nilsson, A. C. Tropper, and D. C. Hanna, "Ytterbiumdoped fiber amplifiers," IEEE J. Quantum Electron., vol. 33, no. 7, pp. 1049-1056, Jul. 1997.

[26] G. P. Agrawal, Applications of Nonlinear Fiber Optics. San Diego, CA: Academic, 2001, ch. 4.

[27] L. Kuznetsova, F. W. Wise, S. Kane, and J. Squier, "Chirped-pulse amplification near the gain-narrowing limit of Yb-doped fiber using a reflection grism compressor," Appl. Phys. B, vol. 88, no. 4, pp. 515-518, 2007.

[28] A. E. Siegman and S. W. Townsend, "Output beam propagation and beam quality from a multimode stable-cavity laser," IEEE J. Quantum Electron., vol. 29, no. 4, pp. 1212-1217, Apr. 1993.

[29] F. O. Ilday, J. Chen, A. Winter, F. X. Kartner, F. W. Wise, O. Shkurikhin, and D. Gapontsev, "Low-noise, high-energy, single-mode, femtosecond fiber laser system," in Proc. Conf. Lasers Electro-Opt. (CLEO 2005), vol. 2, pp. 825-827.

[30] R. P. Scott, C. Langrock, and B. H. Kolner, "High-dynamic-range laser amplitude and phase noise measurement techniques," IEEE J. Quantum Electron., vol. 7, no. 4, pp. 641-655, Jul./Aug. 2001.

[31] M. Tröbs, P. Wessels, and C. Fallnich, "Power- and frequency-noise characteristics of an Yb-doped fiber amplifier and actuators for stabilization," Opt. Express, vol. 13, pp. 2224-2235, 2005.

[32] S. Zhou, L. Kuznetsova, A. Chong, and F. W. Wise, "Compensation of nonlinear phase shifts with third-order dispersion in short-pulse fiber amplifiers," Opt. Express, vol. 13, pp. 4869-4877, 2005.

[33] L. Shah, Z. Liu, I. Hartl, G. Imeshev, G. C. Cho, and M. E. Fermann, "High energy femtosecond $\mathrm{Yb}$ cubicon fiber amplifier," Opt. Express, vol. 13, pp. 4717-4722, 2005.

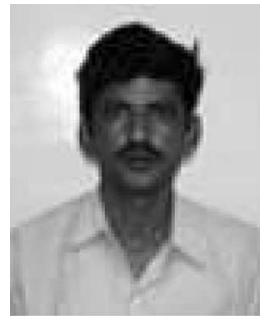

Pranab K. Mukhopadhyay received the Ph.D. degree from Devi Ahilya University, Indore, India, in 2006.

He was engaged in research under the guidance of Dr. P. K. Gupta, Head, Biomedical Application of Laser Division, Raja Ramanna Centre for Advanced Technology (RRCAT), Indore. During 2007-2008, he has been a Postdoctoral Research Fellow in the Ultrafast Optics Laboratory, Bilkent University, Ankara, Turkey, under the guidance of Prof. F. Ö. Ilday, with special focus on ultrafast fiber lasers and amplifiers. He is currently a Scientific Officer at the Solid State Laser Division, RRCAT, where he is engaged in research in the field of diode-pumped solid-state lasers and frequency conversions.

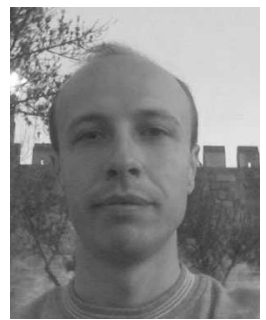

Kıvanc Özgören was born in Ankara, Turkey, in 1980. He received the B.S. degrees in mechanical engineering and physics in 2003 and the M.S. degree in physics in 2005, both from Middle East Technical University, Ankara. He is currently working toward the $\mathrm{Ph} . \mathrm{D}$. degree in materials science and nanotechnology at Bilkent University, Ankara, under the guidance of Prof. F. Ö. Ilday.

He was an Intern at Turk Traktor, Ltd., and ASELSAN Ltd. He completed his compulsory military service in 2006 . His current research interests include the development of high-power ultrafast fiber oscillators and amplifiers.

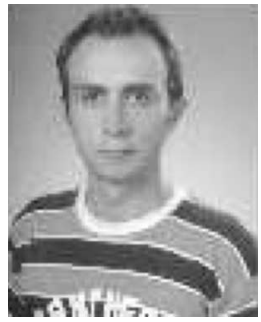

I. Levent Budunoğlu was born in Balikesir, Turkey, in 1982.

He received the B.S. degree in physics in 2006 from Bilkent University, Ankara, Turkey, where he is currently working toward the Ph.D. degree in the Physics Department. His current research interests include noise processes in fiber lasers and development of fiber lasers for optical frequency metrology applications.

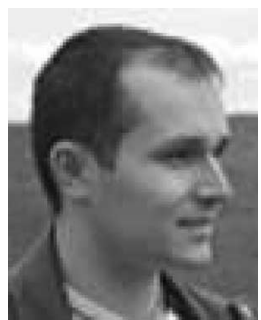

F. Ömer İlday was born in Istanbul, Turkey, in 1976. $\mathrm{He}$ received the B.S. degree in theoretical physics from Bogazici University, Istanbul, in 1998, and the $\mathrm{Ph}$.D. degree in applied physics from Cornell University, Ithaca, NY, in 2003.

He was initially a Postdoctoral Scientist and then a Research Scientist in the Department of Electrical Engineering and Computer Science, Massachusetts Institute of Technology, Cambridge, from 2003 to 2006. He is currently an Assistant Professor of physics at Bilkent University, Ankara, Turkey, since

2007

Dr. Ilday is a member of the Optical Society of America. He serves on the Editorial Board of Optics Letters. 